\title{
A NOTE ON LATTICE VARIANT OF THRESHOLDNESS OF BOOLEAN FUNCTIONS
}

\author{
ESZTER K. HORVÁTH, BRANIMIR ŠEŠELJA, AND ANDREJA TEPAVČEVIĆ \\ Received 02 January, 2015
}

\begin{abstract}
Lattice induced threshold function is a Boolean function determined by a particular linear combination of lattice elements. We prove that every isotone Boolean function is a lattice induced threshold function and vice versa. We give the generalization of this result to Boolean functions on a $k$-element set.
\end{abstract}

2010 Mathematics Subject Classification: 06E30

Keywords: lattice induced threshold functions, isotone Boolean functions

\section{INTRODUCTION}

„Threshold functions provide a simple but fundamental model for many questions investigated in electrical engineering, artificial intelligence, game theory and many other areas." (Quotation from [3].) In [10], modeling neurons and political decisions are also mentioned, as application of classical threshold functions. A classical threshold function is a Boolean function $f:\{0,1\}^{n} \rightarrow\{0,1\}$ such that there exist real numbers $w_{1}, \ldots, w_{n}, t$, fulfilling

$$
f\left(x_{1}, \ldots, x_{n}\right)=1 \text { if and only if } \sum_{i=1}^{n} w_{i} \cdot x_{i} \geq t,
$$

where $w_{i}$ is called the weight of $x_{i}$, for $i=1,2, \ldots, n$ and $t$ is a constant called the threshold value. In this paper we define a new but related notion: the so called lattice induced threshold function and we investigate its properties.

Research of the first author is partially supported by the NFSR of Hungary (OTKA), grant numbers K 115518 and K 83219. Supported by the European Union and co-funded by the European Social Fund under the project "Telemedicine-focused research activities on the field of Mathematics, Informatics and Medical sciences" of project number "TÁMOP-4.2.2.A-11/1/KONV-2012-0073".

Research of the second and the third author is partially supported by Ministry of Education, Science and Technological Development of the Republic of Serbia, Grant No. 174013.

Research of all authors is partially supported by the Provincial Secretariat for Science and Technological Development, Autonomous Province of Vojvodina, grant "Ordered structures and applications". 


\subsection{Historical background}

For the historical as well as for the basic mathematical background we recommend the books $[3,5,10,11]$. For some algebraic aspects of classical threshold functions, we list relevant algebraic papers from several areas of algebra. In [1] the authors reveal the connection between classical threshold functions and fundamental ideals of group-rings. Paper [8] determines the invariance groups of threshold functions. The topic of monotone Boolean functions has constantly been investigated. For the recent results see e.g. [2, 6,9]. Paper [7] proves that classical threshold functions cannot be characterized by a finite set of standard or generalized constraints.

\subsection{Motivation}

Isotone Boolean functions constitute a clone; threshold functions are not closed under superposition, see Theorem 9.2 in [3], so they do not constitute a clone. It is easy to see that threshold functions with positive weights and a threshold value are isotone. However, an isotone Boolean function is not necessarily threshold, e.g. $f=x \cdot y \vee w \cdot z$ is isotone, but not a threshold function. To see this, it is enough to consider its invariance group, which is the following:

$$
D 8=\{(),(1324),(12)(34),(1423),(12),(34),(13)(24),(14)(23)\},
$$

however by [8] the invariance group of any threshold function is a direct product of symmetric groups.

Therefore, our aim is to generalize threshold functions in the framework of lattice valued functions, and then, in particular, to obtain a characterization of all isotone Boolean functions and to represent them by particular linear combinations.

\subsection{Outline}

In Preliminaries, we present properties of lattice valued functions, Boolean functions and classical threshold functions.

In Section 3 we define threshold functions induced by complete lattices; these are Boolean functions determined by a particular linear combination of lattice elements. We prove that every isotone Boolean function is a lattice induced threshold function and vice versa. In Section 4 we give a generalization of results for isotone functions on $k$-element set. In Section 5 we present some examples and further observations.

\section{PRELIMINARIES}

\subsection{Order, lattices}

Our basic notion is a partially ordered set, ordered set, poset, denoted by $(P, \leq)$, where $\leq$ is an order on a set $P$. If a poset is a lattice, then it is denoted by $(L, \leq)$, with the meet and the join of $a, b \in L$ being $a \wedge b$ and $a \vee b$ respectively. The bottom and the top of a poset, if they exist, are 0 and 1 , respectively. If $(P, \leq)$ is a poset, 
then we denote by $\bigwedge M$ and $\bigvee M$ the meet and the join of $M \subseteq P$ respectively, if they exist. An up-set (semi-filter) on a poset $(P, \leq)$ is a subset $F$ of $P$ fulfilling the property: if $x \in F$ and $x \leq y$, then $y \in F$.

We deal with complete lattices and free distributive lattices with $n$ generators. We also use finite Boolean lattices, represented by all $n$-tuples of 0 and 1, ordered componentwise, and denoted by $\left(\{0,1\}^{n}, \leq\right)$.

\subsection{Boolean functions; threshold functions}

A Boolean function is a mapping $f:\{0,1\}^{n} \rightarrow\{0,1\}, n \in \mathbb{N}$.

The domain $\{0,1\}^{n}$ of a Boolean function is usually ordered componentwise, with respect to the natural order $0 \leq 1:\left(x_{1}, x_{2}, \ldots, x_{n}\right) \leq\left(y_{1}, y_{2}, \ldots, y_{n}\right)$ if and only if for every $i \in\{1, \ldots, n\}, x_{i} \leq y_{i}$.

As it is known, the poset $\left(\{0,1\}^{n}, \leq\right)$ is a Boolean lattice. Moreover, every finite Boolean lattice with $n$ atoms is isomorphic to this one.

A Boolean function $f:\{0,1\}^{n} \rightarrow\{0,1\}$ is isotone (order preserving, positive, as in [3]), if for every $x, y \in\{0,1\}^{n}$, from $x \leq y$, it follows that $f(x) \leq f(y)$.

The following is easy to check.

Lemma 1. The set $F \subseteq\{0,1\}^{n}$ is an up-set on $\left(\{0,1\}^{n}, \leq\right)$ if and only if a Boolean function $f$ defined by

$$
f(x)=1 \text { if and only if } x \in F
$$

is isotone.

As mentioned in Introduction, a threshold function is a Boolean function $f$ : $\{0,1\}^{n} \rightarrow\{0,1\}$ such that there exist real numbers $w_{1}, \ldots, w_{n}, t$, fulfilling

$$
f\left(x_{1}, \ldots, x_{n}\right)=1 \text { if and only if } \sum_{i=1}^{n} w_{i} \cdot x_{i} \geq t,
$$

where $w_{i}$ are called the weights of $x_{i}$, for $i=1,2, \ldots, n$ and $t$ is a constant called the threshold value.

\section{THRESHOLD FUNCTIONS INDUCED B Y COMPLETE LATTICES}

In this section we introduce threshold functions induced by complete lattices, and we use them for investigating isotone Boolean functions and their representation.

We deal with Boolean functions over the Boolean lattice $\left(\{0,1\}^{n}, \leq\right)$, and in addition we use a complete lattice $L$ in which the bottom and the top are (also) denoted by 0 and 1 respectively; however, it is clear from the context whether 0 (1) is a component in some $\left(x_{1}, \ldots, x_{n}\right) \in\{0,1\}^{n}$, or it is from $L$. 
For $x \in\{0,1\}$, and $w \in L$, we define a mapping $L \times\{0,1\}$ into $L$ denoted by ".", as follows:

$$
w \cdot x:=\left\{\begin{array}{lll}
w, & \text { if } & x=1 \\
0, & \text { if } & x=0
\end{array}\right.
$$

A function $f:\{0,1\}^{n} \rightarrow\{0,1\}$ is a lattice induced threshold function, if there is a complete lattice $L$ and $w_{1}, \ldots, w_{n}, t \in L$, such that

$$
f\left(x_{1}, \ldots, x_{n}\right)=1 \text { if and only if } \bigvee_{i=1}^{n}\left(w_{i} \cdot x_{i}\right) \geq t .
$$

Proposition 1. Every lattice induced threshold function is isotone.

Proof. Let $L$ be a complete lattice, let $w_{1}, \ldots, w_{n}, t \in L$, and $f:\{0,1\}^{n} \rightarrow\{0,1\}$ a lattice induced threshold function.

Let $\left(x_{1}, x_{2}, \ldots, x_{n}\right) \leq\left(y_{1}, y_{2}, \ldots, y_{n}\right)$. Then, for every $i$, we have $w_{i} \cdot x_{i} \leq w_{i} \cdot y_{i}$, by the definition (3.1). Hence,

$$
\bigvee_{i=1}^{n}\left(w_{i} \cdot x_{i}\right) \leq \bigvee_{i=1}^{n}\left(w_{i} \cdot y_{i}\right) .
$$

Therefore, if $f\left(x_{1}, \ldots, x_{n}\right)=1$, then

$$
\bigvee_{i=1}^{n}\left(w_{i} \cdot x_{i}\right) \geq t \text {, and hence } \bigvee_{i=1}^{n}\left(w_{i} \cdot y_{i}\right) \geq t \text {. }
$$

This implies $f\left(y_{1}, \ldots, y_{n}\right)=1$ and we obtain

$$
f\left(x_{1}, \ldots, x_{n}\right) \leq f\left(y_{1}, \ldots, y_{n}\right),
$$

which proves that $f$ is an isotone function.

Theorem 1. Every isotone Boolean function is a lattice induced threshold function.

Proof. We prove that for every $n \in \mathbb{N}$, there is a lattice $L$ such that every isotone Boolean function is a lattice induced threshold function over $L$.

Let $n \in \mathbb{N}$. We take $L$ to be a free distributive lattice with $n$ generators $w_{1}, w_{2}, \ldots$, $w_{n}$ (the join and meet of empty set of generators are also counted here, as the bottom and the top of $L$, respectively). Recall that every element in a free distributive lattice can be uniquely represented in a "conjunctive normal form" by means of generators (i.e., every element is a meet of elements of the type $\bigvee_{i \in J} w_{j}$, where $J \subseteq\{1, \ldots, n\}$,) see e.g., [4].

Therefore, for $x, y \in L$, if $x=\bigwedge_{k=1}^{p} \bigvee_{j \in I_{k}} w_{j}$ and $y=\bigwedge_{k=1}^{l} \bigvee_{s \in J_{k}} w_{s}$, then $x \leq y$ if and only if for every $u \in\{1, \ldots, l\}$ there is $k \in\{1, \ldots, p\}$ such that $I_{k} \subseteq J_{u}$. $(*)$ 
Let $f:\{0,1\}^{n} \rightarrow\{0,1\}$ be an isotone Boolean function. Let $F$ be the corresponding up-set on $\{0,1\}^{n}$ (according to Lemma 1). Further, let $m_{1}, \ldots, m_{p}$ be minimal elements of this up-set. Let $I_{1}, \ldots, I_{p}$ be subsets of $\{1,2, \ldots, n\}$, i.e., sets of indices, such that $i \in I_{k}$ if and only if $i$-th coordinate of $m_{k}$ is equal to 1 .

For the threshold $t \in L$ associated to the given function $f$ we take

$$
t=\bigwedge_{k=1}^{p} \bigvee_{j \in I_{k}} w_{j}
$$

Now, we prove that

$$
f\left(x_{1}, \ldots, x_{n}\right)=1 \text { if and only if } \bigvee_{i=1}^{n}\left(w_{i} \cdot x_{i}\right) \geq t .
$$

Indeed, from $f\left(x_{1}, \ldots, x_{n}\right)=1$, it follows that there is a minimal element $m_{l}$ in the corresponding up-set, such that $\left(x_{1}, \ldots, x_{n}\right) \geq m_{l}$. Hence,

$$
\bigvee_{i=1}^{n}\left(w_{i} \cdot x_{i}\right) \geq \bigvee_{j \in I_{l}} w_{j} \geq \bigwedge_{k=1}^{p} \bigvee_{j \in I_{k}} w_{j}=t .
$$

Now we suppose that

$$
\bigvee_{i=1}^{n}\left(w_{i} \cdot x_{i}\right) \geq \bigwedge_{k=1}^{p} \bigvee_{j \in I_{k}} w_{j}
$$

for an ordered n-tuple $\left(x_{1}, \ldots, x_{n}\right)$. Let $I \subseteq\{1, \ldots n\}$ be the set of indices such that $x_{i}=1$ if and only if $i \in I$. We prove that there is $s \in\{1, \ldots p\}$ such that $I_{s} \subseteq I$. This follows directly from the above mentioned $(*)$ property of the free distributive lattice with $n$ generators $w_{1}, \ldots, w_{n}$.

Now, it follows that $\left(x_{1}, \ldots, x_{n}\right) \geq\left(y_{1}, \ldots, y_{n}\right)$, where $y_{i}=1$ if and only if $i \in I_{s}$. Therefore,

$$
\bigvee_{i=1}^{n}\left(w_{i} \cdot y_{i}\right) \geq t, \text { and } f\left(y_{1}, \ldots, y_{n}\right)=1 .
$$

This finally implies $f\left(x_{1}, \ldots, x_{n}\right)=1$.

Remark 1. In the previous proposition it is proved not only that every isotone $n$-ary Boolean function is a lattice induced threshold function, but also that the corresponding lattice in each case can be the free distributive lattice with $n$ generators.

Remark 2. If we want to induce all isotone function with one distributive lattice, then clearly no lattice with smaller cardinality than the free distributive lattice with $n$ generators is appropriate, since different elements of the free distributive lattice provide different isotone functions.

Here we investigate lattice valued functions on finite Boolean lattices. 


\subsection{Representation of isotone lattice valued Boolean functions by cuts}

A function $f:\{0,1\}^{n} \rightarrow L$, where $L$ is a complete lattice, is a lattice valued ( $L$-valued) Boolean function.

For $f:\{0,1\}^{n} \rightarrow L$ and $p \in L$, a cut set (cut) $f_{p}$ is a subset of $\{0,1\}^{n}$ :

$$
f_{p}=\left\{x \in\{0,1\}^{n} \mid f(x) \geq p\right\} .
$$

A characteristic function of the cut $f_{p}$ is an ordinary Boolean function and we denote it by the same symbol $f_{p}$.

The following lemma is straightforward.

Lemma 2. Let $B$ be a Boolean lattice and $\mu: B \rightarrow L$ an $L$-valued Boolean function. Then $\mu$ is isotone if and only if all the cuts of $\mu$ are up-sets on $B$.

In this part, we represent isotone $L$-valued functions on $B$ in the framework of cut sets and lattice induced threshold functions.

Our main tool here is a particular $L$-valued Boolean function, defined as follows.

Let $B=\left(\{0,1\}^{n}, \leq\right), n \in \mathbb{N}$, let $L_{D}$ a free distributive lattice with $n$ generators $w_{1}, \ldots, w_{n}$ and $\bar{\beta}: B \rightarrow L_{D}$, an $L_{D}$-valued function on $B$ defined in the following way: for $x=\left(x_{1}, \ldots, x_{n}\right) \in B$

$$
\bar{\beta}(x)=\bigvee_{i=1}^{n}\left(w_{i} \cdot x_{i}\right),
$$

where the function "." is defined by (3.1).

By the definition, $\bar{\beta}$ is uniquely (up to a permutation of generators $w_{i}$ ) determined by a finite Boolean lattice $B=\left(\{0,1\}^{n}, \leq\right)$, i.e., by a positive integer $n$.

A connection of isotone functions and cuts is presented in Lemma 2. Next we prove that in this framework, $\bar{\beta}$ can be considered as the main representative of all isotone $L$-valued functions on $B$.

Theorem 2. Every up-set of a finite Boolean lattice $B=\left(\{0,1\}^{n}, \leq\right), n \in \mathbb{N}$, is a cut of $\bar{\beta}$.

Proof. Let $B=\left(\{0,1\}^{n}, \leq\right)$ be a finite Boolean lattice. By Theorem 1, every isotone Boolean function with $n$ variables is a lattice induced threshold function over a free distributive lattice $L_{D}$ with $n$ generators. By Lemma 1 , every up-set on $B$ is (as a characteristic function) an isotone Boolean function $f:\{0,1\}^{n} \rightarrow\{0,1\}$. This means that there are elements $w_{1}, \ldots, w_{n} \in L_{D}$ (generators), such that

$$
f\left(x_{1}, \ldots, x_{n}\right)=1 \text { if and only if } \bigvee_{i=1}^{n}\left(w_{i} \cdot x_{i}\right) \geq t \text {, for some } t \in L_{D} \text {. }
$$

Thereby, by (3.4),

$$
f\left(x_{1}, \ldots, x_{n}\right)=1 \text { if and only if } \bar{\beta}(x) \geq t \text { if and only if } x \in \bar{\beta}_{t} .
$$


Hence the up-set whose characteristic function is $f$, coincides with the cut $\bar{\beta}_{t}$ of $\bar{\beta}$.

By Lemma 2, we have the following obvious consequence of Theorem 2.

Corollary 1. Lattice valued function $\bar{\beta}$ defined by (3.4) is isotone. Moreover, the collection of cuts of every $L$-valued isotone function on $B$ (for any $L$ ) is contained in the collection of cuts of $\bar{\beta}$.

\section{Generalization: isotone fUnCtions On The $k$-Element SET}

In this part we investigate isotone functions on a $k$-element set $\{1,2, \ldots, k\}$. Generalizing previously presented techniques, we introduce multi-value threshold functions induced by complete lattices and use them for representing the mentioned isotone functions.

Let $L$ be a complete lattice with the bottom element 0 and top element 1 . For $x \in\{1, \ldots, k\}$, and $w^{1}, \ldots, w^{k} \in L$, we define a mapping

$$
\left\{w^{1}, \ldots, w^{k}\right\} \times\{1, \ldots, k\} \rightarrow L
$$

denoted by ".", as follows:

$$
w^{j} \cdot x:=\left\{\begin{array}{lll}
w^{j}, & \text { if } & j \leq x, \\
0 & \text { otherwise. }
\end{array}\right.
$$

Now, $f:\{1, \ldots, k\}^{n} \rightarrow\{1, \ldots, k\}$ is a lattice induced multi-value threshold function, if there is a complete lattice $L$ and $w_{1}^{1}, \ldots, w_{1}^{k}, \ldots, w_{n}^{1}, \ldots, w_{n}^{k}, t_{i_{1}}, \ldots, t_{i_{p}} \in$ $L$, where $i_{1}, \ldots, i_{p} \in\{1, \ldots k\}$ and from $c<d$ it follows that $i_{c}<i_{d}{ }^{1}$, such that for $r \in\{1, \ldots, k\}$,

$$
\begin{gathered}
f\left(x_{1}, \ldots, x_{n}\right)=r \text { if and only if } \\
\bigvee_{i=1}^{n} \bigvee_{j=1}^{k}\left(w_{i}^{j} \cdot x_{i}\right) \in \uparrow t_{r}, \text { for } r=i_{p} \text { and } \\
\bigvee_{i=1}^{n} \bigvee_{j=1}^{k}\left(w_{i}^{j} \cdot x_{i}\right) \in \uparrow t_{r} \backslash\left(\bigcup_{h>r} \uparrow t_{h}\right), \text { for } r<i_{p} .
\end{gathered}
$$

Proposition 2. Every lattice induced multi-value threshold function is isotone.

Proof. Let $L$ be a complete lattice and $w_{1}^{1}, \ldots, w_{1}^{k}, \ldots, w_{n}^{1}, \ldots, w_{n}^{k}, t_{i_{1}}, \ldots, t_{i_{p}} \in L$, where $i_{1}, \ldots, i_{p} \in\{1, \ldots k\}$

such that for $r \in\{1, \ldots, k\}$,

$$
\begin{gathered}
f\left(x_{1}, \ldots, x_{n}\right)=r \text { if and only if } \\
\bigvee_{i=1}^{n} \bigvee_{j=1}^{k}\left(w_{i}^{j} \cdot x_{i}\right) \in \uparrow t_{r}, \text { for } r=i_{p} \text { and } \\
\bigvee_{i=1}^{n} \bigvee_{j=1}^{k}\left(w_{i}^{j} \cdot x_{i}\right) \in \uparrow t_{r} \backslash\left(\bigcup_{h>r} \uparrow t_{h}\right), \text { for } r<i_{p}
\end{gathered}
$$

\footnotetext{
${ }^{1}$ Hence, $i_{p}$ is the greatest element of $i_{1}, \ldots, i_{p}$
} 
is a lattice induced multi-value threshold function.

Let $\left(x_{1}, x_{2}, \ldots, x_{n}\right) \leq\left(y_{1}, y_{2}, \ldots, y_{n}\right)$. Then, for every $i \in\{1, \ldots, k\}$

$$
\bigvee_{j=1}^{k}\left(w_{i}^{j} \cdot x_{i}\right) \leq \bigvee_{j=1}^{k}\left(w_{i}^{j} \cdot y_{i}\right)
$$

by the definition (4.1) of the mapping ".". Hence,

$$
\bigvee_{i=1}^{n} \bigvee_{j=1}^{k}\left(w_{i}^{j} \cdot x_{i}\right) \leq \bigvee_{i=1}^{n} \bigvee_{j=1}^{k}\left(w_{i}^{j} \cdot y_{i}\right)
$$

Therefore, if $f\left(x_{1}, \ldots, x_{n}\right)=r$, then

$$
\bigvee_{i=1}^{n} \bigvee_{j=1}^{k}\left(w_{i}^{j} \cdot x_{i}\right) \geq t_{r} \text { and hence } \bigvee_{i=1}^{n} \bigvee_{j=1}^{k}\left(w_{i}^{j} \cdot y_{i}\right) \geq t_{r}
$$

This implies $\bigvee_{i=1}^{n} \bigvee_{j=1}^{k}\left(w_{i}^{j} \cdot y_{i}\right) \in\left(\uparrow t_{r} \backslash\left(\bigcup_{h>r} \uparrow t_{h}\right)\right) \bigcup\left(\bigcup_{h>r} \uparrow t_{h}\right)$, hence, either $f\left(y_{1}, \ldots, y_{n}\right)=r$ or $f\left(y_{1}, \ldots, y_{n}\right)>r$, in either case $f\left(x_{1}, \ldots, x_{n}\right) \leq f\left(y_{1}, \ldots, y_{n}\right)$.

Theorem 3. Every isotone function on the $k$-element set is a lattice induced multivalue threshold function.

Proof. We prove that for any natural number $n$, and $k>2$, there is a lattice $L$, such that every $n$-ary isotone function on the $k$-element set is a lattice induced multi-value threshold function.

This lattice $L$ is a free distributive lattice with $k \cdot n$ generators $w_{1}^{1}, \ldots, w_{1}^{k}$, $\ldots, w_{n}^{1}, \ldots, w_{n}^{k}$. In some instances we denote the set of generators by $\left\{w_{1}^{1}, \ldots\right.$, $\left.w_{1}^{k}, \ldots, w_{n}^{1}, \ldots, w_{n}^{k}\right\}$ and in other by $\left\{w_{j} \mid j \in\{1, \ldots, k \cdot n\}\right\}$.

If $x, y \in L, x=\bigwedge_{u=1}^{p} \bigvee_{j \in I_{u}} w_{j}$ and $y=\bigwedge_{u=1}^{l} \bigvee_{s \in J_{u}} w_{s}$, then $x \leq y$ if and only if for every $u \in\{1, \ldots, p\}$ there is $v \in\{1, \ldots, l\}$ such that $I_{u} \subseteq J_{v}$.

Let $f:\{1, \ldots, k\}^{n} \rightarrow\{1, \ldots, k\}$ be an isotone function on the $k$-element set. Let $r$ be an element $1 \leq r \leq k$ and let $F_{r}$ be the up-set corresponding to $r$, i.e. $F_{r}=$ $f^{-1}(\uparrow r)$. Observe that in case no element is mapped by $f$ to some $a \in\{1, \ldots, k\}$, then $F_{a}=F_{a+1}$. Further, let $m_{r, 1}, \ldots, m_{r, p_{r}}$ be minimal elements of this up-set, in case $F_{r} \backslash \bigcup_{h>r} F_{h}$ is not empty. For $s \in\left\{1, \ldots, p_{r}\right\}$, let $m_{r, s}=\left(m_{1, r, s}, \ldots, m_{n, r, s}\right)$.

For the thresholds $t_{i_{1}}, \ldots, t_{i_{p}} \in L$, where $i_{1}, \ldots, i_{p} \in\{1, \ldots k\}$ are associated to the given function $f$, we take

$$
t_{r}=\bigwedge_{s=1}^{p_{r}} \bigvee_{i=1}^{n} \bigvee_{j=1}^{k}\left(w_{i}^{j} \cdot m_{i, r, s}\right) \quad \text { for } r \in\{1, \ldots, k\} \text { for which } F_{r} \backslash \bigcup_{h>r} F_{h} \neq \varnothing
$$

Now we show that 


$$
\begin{gathered}
f\left(x_{1}, \ldots, x_{n}\right)=r \text { if and only if } \\
\bigvee_{i=1}^{n} \bigvee_{j=1}^{k}\left(w_{i}^{j} \cdot x_{i}\right) \in \uparrow t_{r}, \text { for } r=i_{p} \text { and } \\
\bigvee_{i=1}^{n} \bigvee_{j=1}^{k}\left(w_{i}^{j} \cdot x_{i}\right) \in \uparrow t_{r} \backslash\left(\bigcup_{h>r} \uparrow t_{h}\right), \text { for } r<i_{p}
\end{gathered}
$$

Suppose that $f\left(x_{1}, \ldots, x_{n}\right)=r$, for $r=i_{p}$. Indeed, from $f\left(x_{1}, \ldots, x_{n}\right) \geq r$, it follows that there is a minimal element $m_{r, l_{r}}$ in the corresponding up-set, such that $\left(x_{1}, \ldots, x_{n}\right) \geq m_{r, l_{r}}=\left(m_{1, r, l_{r}}, \ldots, m_{n, r, l_{r}}\right)$. Hence,

$$
\bigvee_{i=1}^{n} \bigvee_{j=1}^{k}\left(w_{i}^{j} \cdot x_{i}\right) \geq \bigvee_{i=1}^{n} \bigvee_{j=1}^{k}\left(w_{i}^{j} \cdot m_{1, r, l_{r}}\right) \geq \bigwedge_{s=1}^{p_{r}} \bigvee_{i=1}^{n} \bigvee_{j=1}^{k}\left(w_{i}^{j} \cdot m_{i, r, v_{r}}\right)=t_{r}
$$

On the other hand, let $\bigvee_{i=1}^{n} \bigvee_{j=1}^{k}\left(w_{i}^{j} \cdot x_{i}\right) \in \uparrow t_{r}$, for $r=i_{p}$. Then, $\bigvee_{i=1}^{n} \bigvee_{j=1}^{k}\left(w_{i}^{j} \cdot x_{i}\right) \geq \bigwedge_{s=1}^{p_{r}} \bigvee_{i=1}^{n} \bigvee_{j=1}^{k}\left(w_{i}^{j} \cdot m_{i, r, v_{r}}\right)=t_{r}$. By the definition of the order $\leq$ in the free distributive lattice, it follows that there is an $m_{r, l_{r}}$, such that $\bigvee_{i=1}^{n} \bigvee_{j=1}^{k}\left(w_{i}^{j} \cdot x_{i}\right) \geq \bigvee_{i=1}^{n} \bigvee_{j=1}^{k}\left(w_{i}^{j} \cdot m_{1, r, l_{r}}\right)$, and $\left(x_{1}, \ldots, x_{n}\right) \geq m_{r, l_{r}}=$ $\left(m_{1, r, l_{r}}, \ldots, m_{n, r, l_{r}}\right)$ and hence $f\left(x_{1}, \ldots, x_{n}\right)=r$.

Now, suppose that

$f\left(x_{1}, \ldots, x_{n}\right)=r$ for $r<i_{p}$. We have that $F_{r} \backslash \bigcup_{h>r} F_{h} \neq \varnothing$.

As above, from $f\left(x_{1}, \ldots, x_{n}\right) \geq r$, it follows that there is a minimal element $m_{r, l_{r}}$ in the corresponding up-set, such that $\left(x_{1}, \ldots, x_{n}\right) \geq m_{r, l_{r}}=\left(m_{1, r, l_{r}}, \ldots, m_{n, r, l_{r}}\right)$. Hence,

$$
\bigvee_{i=1}^{n} \bigvee_{j=1}^{k}\left(w_{i}^{j} \cdot x_{i}\right) \geq \bigvee_{i=1}^{n} \bigvee_{j=1}^{k}\left(w_{i}^{j} \cdot m_{1, r, l_{r}}\right) \geq \bigwedge_{s=1}^{p_{r}} \bigvee_{i=1}^{n} \bigvee_{j=1}^{k}\left(w_{i}^{j} \cdot m_{i, r, v_{r}}\right)=t_{r}
$$

and

$$
\bigvee_{i=1}^{n} \bigvee_{j=1}^{k}\left(w_{i}^{j} \cdot x_{i}\right) \in \uparrow t_{r} \backslash\left(\bigcup_{h>r} \uparrow t_{h}\right) .
$$

Suppose now that $\left(x_{1}, \ldots, x_{n}\right)$ is an element such that

$$
\bigvee_{i=1}^{n} \bigvee_{j=1}^{k}\left(w_{i}^{j} \cdot x_{i}\right) \in \uparrow t_{r} \backslash\left(\bigcup_{h>r} \uparrow t_{h}\right) .
$$

Now $\bigvee_{i=1}^{n} \bigvee_{j=1}^{k}\left(w_{i}^{j} \cdot x_{i}\right) \geq \bigwedge_{s=1}^{p_{r}} \bigvee_{i=1}^{n} \bigvee_{j=1}^{k}\left(w_{i}^{j} \cdot m_{i, r, v_{r}}\right)$. By the arguments above, we have that there is a minimal element in the corresponding up-set, such that $\left(x_{1}, \ldots, x_{n}\right) \geq m_{r, l_{r}}=\left(m_{1, r, l_{r}}, \ldots, m_{n, r, l_{r}}\right)$. Therefore, $f\left(x_{1}, \ldots, x_{n}\right) \geq r$. By the condition $\bigvee_{i=1}^{n} \bigvee_{j=1}^{k}\left(w_{i}^{j} \cdot x_{i}\right) \notin \uparrow t_{h}$, for $h>r$ we obtain that $f\left(x_{1}, \ldots, x_{n}\right) \nsucceq h$, for $h>r$, hence $f\left(x_{1}, \ldots, x_{n}\right)=r$.

Observe that in the case of isotone functions on a $k$-element sets, the analogue properties to those in Remarks 1 and 2 are satisfied. 


\section{SOME EXAMPLES AND OBSERVATIONS}

In this part we discus whether we can get particular isotone Boolean functions using some lattices different than the free distributive ones.

Starting with our approach, it is possible to characterize isotone Boolean functions satisfying some relationships among components by a free distributive lattice with less than $n$ generators and also by factor lattices of the free distributive lattices.

Without getting into detailed elaboration, in the sequel we briefly describe these characterizations.

1. Isotone Boolean functions $f:\{0,1\}^{n} \rightarrow\{0,1\}$ satisfying

$$
f\left(x_{1}, \ldots, 1, \ldots, 0, \ldots, x_{n}\right)=f\left(x_{1}, \ldots, 0, \ldots, 1, \ldots, x_{n}\right)=f\left(x_{1}, \ldots, 1, \ldots, 1, \ldots, x_{n}\right)
$$

for all $x_{k}$ for $k \in\{1, \ldots, n\} \backslash\{i, j\}, i \neq j$, where 1 and 0 are on $i$-th and $j$-th places, can be characterized by the free distributive lattice with $n-1$ generators (where $w_{i}=$ $\left.w_{j}\right)$.

This can be generalized to the case with $k$ components (out of $n$ ) among which at least one is 1 ; a characterization is obtained by the free distributive lattices with $n-k$ generators (with $w_{i_{1}}=\ldots=w_{i_{k}}$ ).

2. Isotone Boolean functions $f:\{0,1\}^{n} \rightarrow\{0,1\}$ satisfying

$$
f\left(x_{1}, \ldots, 0, \ldots, 1, \ldots, x_{n}\right)=f\left(x_{1}, \ldots, 1, \ldots, 1, \ldots, x_{n}\right)
$$

for all $x_{k}$ with $k \in\{1, \ldots, n\} \backslash\{i, j\}, i \neq j$, where 0 and 1 ( 1 and 1 ), are on $i$-th and $j$-th places, respectively; a characterization is obtained by a factor lattice of free distributive lattice (corresponding to the relationship $w_{i}<w_{j}$ ).

Generalizations to isotone Boolean functions with $k$ ordered components can be characterized using factor lattices of free distributive lattices with $n$ generators (where $w_{i_{1}}, \ldots, w_{i_{k}}$ constitute a corresponding poset).

3. Isotone Boolean functions $f:\{0,1\}^{n} \rightarrow\{0,1\}$ satisfying combinations of various relationships of the type 1 . and 2. can be characterized by factor lattices of the free distributive lattices with $n$ generators (which corresponds to distributive lattices freely generated by posets).

Furthermore, it is possible to characterize Boolean functions by various non -distributive lattices with smaller number of elements. We present here some examples.

Example 1. Let $f:\{0,1\}^{3} \rightarrow\{0,1\}$ be a Boolean function, defined by $f(1,1,1)=$ $f(1,1,0)=f(0,1,1)=f(1,0,1)=1$ and $f(x, y, z)=0$ otherwise. This Boolean function can be represented as a lattice-induced threshold function using a lattice $M_{3}$, where $w_{1}, w_{2}, w_{3}$ are atoms of the lattice and $t$ is the top of the lattice.

Similarly, a representation can be obtained for all analogous Boolean functions $f:\{0,1\}^{n} \rightarrow\{0,1\}$ having value 1 for all $\left(x_{1}, \ldots, x_{n}\right)$ with 2 and more coordinates with value 1 , and having value 0 otherwise, by a lattice $M_{n}$, where $w_{1}, \ldots, w_{n}$ are atoms of the lattice and $t$ is the top of the lattice. 
Example 2. Let $f:\{0,1\}^{3} \rightarrow\{0,1\}$ be a Boolean function, defined by $f(0,0,0)=$ 0 and $f(x, y, z)=1$ otherwise. This Boolean function can be represented as a latticeinduced threshold function using a lattice $0 \oplus M_{3}$ ( $M_{3}$ with an additional bottom element 0 ), where $w_{1}, w_{2}, w_{3}$ are co-atoms and $t$ is the atom of the lattice. A similar representation is possible for all analogous Boolean functions $f:\{0,1\}^{n} \rightarrow\{0,1\}$.

By similar constructions, choosing suitable lattices and elements $w_{1}, \ldots, w_{n}, t$ we can represent all particular Boolean functions. However, if we want to make a representation of all Boolean functions $f:\{0,1\}^{n} \rightarrow\{0,1\}$ by one lattice, then the smallest such lattice is the free Boolean lattice with $n$ generators. Clearly, its number of elements coincides with the number of different Boolean functions.

\section{CONCLUSiON}

To sum up, we have introduced a lattice induced threshold function, as a generalization of the class of classical threshold functions. Our motivation was to capture isotonicity of Boolean functions.

The obtained results could be further generalized in several directions. First of all, one could consider lattice functions instead of Boolean ones. Also, one might look for a characterization of the classical threshold functions within the class of lattice induced threshold functions. Furthermore, as mentioned above, with appropriately chosen lattices as co-domain, interesting classes of Boolean functions could be obtained.

\section{REFERENCES}

[1] N. Aizenberg, A. Bovdi, . E. Gergo, and F. E. Geche, "Algebraic aspects of threshold logic," Cybernetics and Systems Analysis, vol. 16, no. 2, pp. 188-193, 1980, doi: 10.1007/BF01069103.

[2] D. Christofides, "Influences of monotone Boolean functions," Discrete Mathematics, vol. 310, no. 8, pp. 1401-1402, 2010, doi: 10.1016/j.disc.2009.12.017.

[3] Y. Crama and P. Hammer, Boolean Functions, Theory, Algorithms, and Application. New York: Cambridge University Press, 2011.

[4] P. Crawley and P. Dilworth, Algebraic Theory of Lattices. Englewood Cliffs, N.J.: Prentice-Hall, 1973.

[5] B. Davey and H. Priestley, Introduction to Lattices and Order. Cambridge University Press, 1992.

[6] M. Grech, "Regular symmetric groups of boolean functions," Discrete Mathematics, vol. 310, no. 21, pp. 2877-2882, 2010, doi: 10.1016/j.disc.2010.06.036.

[7] L. Hellerstein, "On generalized constraints and certificates," Discrete Mathematics, vol. 226, no. 1, pp. 211-232, 2001, doi: 10.1016/S0012-365X(00)00166-7.

[8] E. Horváth, "Invariance groups of threshold functions," Acta Cybern., vol. 11, no. 4, pp. 325-332, 1994.

[9] N. Keller and H. Pilpel, "Linear transformations of monotone functions on the discrete cube," Discrete Mathematics, vol. 309, no. 12, pp. 4210-4214, 2009, doi: 10.1016/j.disc.2008.12.018.

[10] S. Muroga, Threshold logic and its applications. Wiley and Sons Inc., 1971.

[11] C. Sheng, Threshold logic. London: Academic Press, 1969. 
Authors' addresses

\section{Eszter K. Horváth}

Bolyai Institute, University of Szeged, Aradi vértanúk tere 1, Szeged 6720, Hungary

E-mail address: horeszt@math.u-szeged.hu

Branimir Šešelja

University of Novi Sad, Faculty of Science, Trg D. Obradovića 4, 21000 Novi Sad, Serbia

E-mail address: sesel ja@dmi.uns.ac.rs

Andreja Tepavčević

University of Novi Sad, Faculty of Science, Trg D. Obradovića 4, 21000 Novi Sad, Serbia

E-mail address: andreja@dmi.uns.ac.rs 